\title{
A New Class of Integrated Thermal Oscillators with Duty-cycle Output for Application in Thermal Sensors
}

\author{
Y. PAN, F. R. RIEDIJK and J. H. HUIJSING
}

Electrical Engineering Department, Delft University of Technology, P.O. Box 5031, 2600GA Delft (The Netherlands)

\begin{abstract}
A new class of integrated thermal oscillators has been developed by measuring the heating power in the time domain. A duty-cycle output proportional to the heat-transfer coefficient has been obtained to be compatible with microprocessors. Several different structures, which belong to the same family, have been constructed. The converter family can be employed to measure flow velocity, pressure, IR radiation and true RMS with fast response and good linearity. Experimented results for measuring gas-flow velocity are presented. The structures of the oscillator are simple and only a standard bipolar technique is required.
\end{abstract}

\section{Introduction}

The measurement of many physical parameters such as flow, pressure or IR radiation is often based on the use of thermal sensors, which measure the heat transfer between a heated chip and its ambient. A schematic representation of this class of sensors is depicted in Fig. 1. It contains three domains: (i) physical, (ii) thermal and (iii) electrical. In the last decade, three principles have been developed to convert the physical signal into a thermal signal, these include: (i) the measurement of a temperature difference $[1,2]$; (ii) the measurement of a heating power $[3,4]$ and (iii) the measurement of rise and fall times [5].

The measurement of on-chip heating power dissipation is a well-known method. A thermal feedback loop, which is usually constructed by an on-chip heating source, a temperature sensing element and an amplifier, should be used to keep the chip at a constant temperature in order to achieve a linear relation between the heating power and the heat-transfer coefficient. It is expected from previous reports that a linear relation and a fast response can be achieved with a very high loop gain. We found, however, that this result can only be achieved with an ideal thermal feedback loop with one dominant pole caused by the thermal

$0924-4247 / 90 / \$ 3.50$

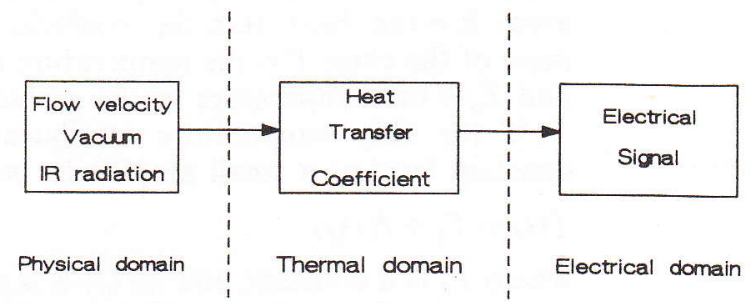

Fig. 1. The three domains of thermal sensors.

capacitance of the chip. In practice, the loop will easily be brought into oscillation by several nonideal effects, such as:

(i) delay of the amplifier and of the heat transfer between the heating element and the temperature sensing element;

(ii) parastic positive feedback loops;

(iii) $50 \mathrm{~Hz}$ background interference;

(vi) thermal noise in the input of the amplifier.

To overcome these disadvantages, a novel thermal sensor for measuring flow velocity has been recently reported by the present authors [6]. In this configuration, the loop has deliberately been forced into oscillation by a high loop gain and positive feedback at small temperature oscillation amplitude. The average heating power is measured in the time domain. A linear relation between duty cycle, heating power and heat-transfer coefficient can therefore be achieved.

The present paper describes the extension of the above structure to a converter family with improved performance and flexibility for application in smart sensors based on the measurement of thermal power. The principle of power measurement in the time domain is discussed in Section 2, the members of the converter family are distinguished in Section 3, and some results for measuring gas-flow velocity are presented in Section 4.

\section{Principle of Measurement}

A basic monolithic integrated thermal sensor can be realized by an on-chip heating source and

(C) Elsevier Sequoia/Printed in The Netherlands 
a temperature sensor. The on-chip heating power is dissipated mainly through two mechanisms in a chip with a well designed substrate contact: ture;

(i) through an increase in the chip tempera-

(ii) through compensation of the heat convection between the heated chip and ambient.

The power balance equation can be written as:

$P_{\mathrm{h}}=C_{\mathrm{th}} m \frac{\mathrm{d} T}{\mathrm{~d} t}+A_{\mathrm{c}} h\left(T-T_{\mathrm{a}}\right)$

where $P_{\mathrm{h}}=$ the heating power; $C_{\mathrm{th}}=$ the thermal capacitance of the chip; $A_{\mathrm{c}}=$ the chip surface area; $h=$ the heat transfer coefficient; $m=$ the mass of the chip; $T=$ the temperature of the chip; and $T_{\mathrm{a}}=$ the temperature of the ambient.

If the chip temperature oscillates around a constant level at a small amplitude, we obtain

$T(t)=T_{0}+\Delta T(t)$

where $T_{0}$ is a constant and $\Delta T(t)$ is a small signal periodic oscillation function of time. Equation (1) can be rewritten as

$P_{\mathrm{h}}(t)=C_{\mathrm{th}} m\left(\frac{\mathrm{d} \Delta T(t)}{\mathrm{d} t}\right)+A_{\mathrm{c}} h\left(T_{0}+\Delta T(t)-T_{\mathrm{a}}\right)$

Both sides of eqn. (3) are averaged in the time domain, then we obtain

$$
\begin{aligned}
P_{\mathrm{ave}}= & C_{\mathrm{th}} m\left(\frac{\mathrm{d} \Delta T(t)}{\mathrm{d} t}\right)_{\mathrm{ave}} \\
& +A_{\mathrm{c}} h\left(T_{0}+(\Delta T(t))_{\mathrm{ave}}-T_{\mathrm{a}}\right)
\end{aligned}
$$

where the subscript 'ave' means the average value in one oscillation period. It is obvious that for a periodic oscillation function with a sufficiently small amplitude around the zero point,

$\left(\frac{\mathrm{d} \Delta T(t)}{\mathrm{d} t}\right)_{\mathrm{ave}} \approx(\Delta T(t))_{\mathrm{ave}} \approx 0$

Therefore, we achieve

$$
P_{\text {ave }} \approx A_{\mathrm{c}} h\left(T_{0}-T_{\mathrm{a}}\right)
$$

Equation (6) demonstrates clearly that the average heating power is linearly related to the heattransfer coefficient with constant average chip temperature. In addition, also a very short response time can be achieved because of the very high loop gain. Such disadvantages as nonlinearity and long response time caused in previous stable-state heating power measurements no longer exist.

Although the chip temperature could oscillate naturally at a high loop gain because of the time delay of the heat transfer between the on-chip heating source and the temperature sensing element, a well-defined pulse-modulated block wave is usually preferred in practical applications which can be transmitted to a microprocessor directly.
This can lead to the fabrication of a so-called 'smart sensor'. Several configurations have been developed to provide a flexible and well-defined output signal. They are discussed in the next section.

\section{Converter Family}

Several structures have been developed to force the thermal feedback loop into oscillation with a block wave output. They are:

(i) free-running thermal oscillator with positive feedback as published before by the authors [6];

(ii) thermal oscillator with positive feedback, synchronized by an external clock;

(iii) sigma-delta thermal modulator with an additional clocked flip-flop.

These three structures are discussed below.

\subsection{Free-running Thermal Oscillator with Positive Feedback}

The basic sensor circuit is depicted in Fig. 2 (removing the external clock $\mathrm{V}_{\mathrm{c}}$ and resistor $\mathbf{R}_{\mathrm{cl}}$ ). It contains a diode $D_{1}$ for measuring the chip temperature and an SP resistor $\mathbf{R}_{h}$ for heating the chip. The diode $D_{2}$ on another chip is utilised to measure the ambient temperature and resistors $R_{1}$ and $R_{2}$ are used to provide the bias current for $D_{1}$ and $\mathrm{D}_{2}$. The emitter/base offset is set up by the reference resistor $R_{r}$, which makes the sensor chip operate at an overheat of the ambient. A power transistor $Q_{h}$ with a collector bias resistor $R_{c}$ is employed as a switch. A comparator with a positive feedback resistor $R_{\mathbf{f}}$ forces the thermal feedback loop to function as a block-wave oscillator.

In the initial state, the output of the comparator is set high and the heating resistor will begin to heat the chip. As a result, the temperature of the chip will increase and $V_{1}$ will decrease until the output of the comparator switches low. Then the chip temperature will decrease due to heat exchange with the ambient until the output of the

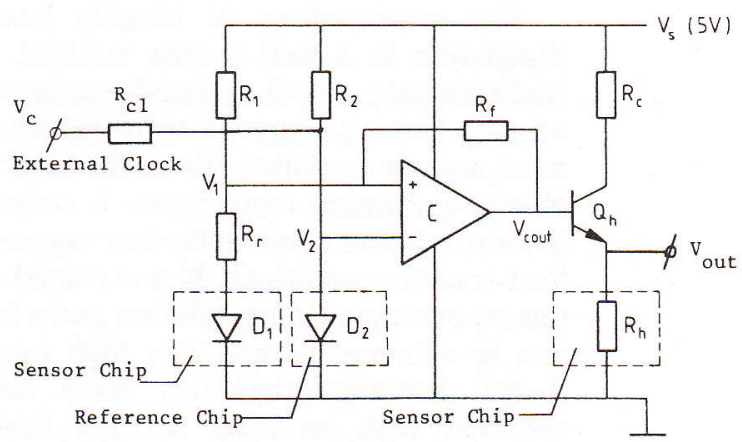

Fig. 2. Basic oscillation circuits. 


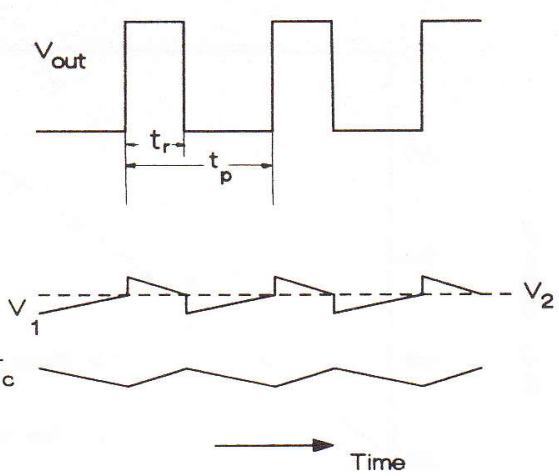

Fig. 3. Waveforms of the basic oscillator.

oscillation of the comparator is high again. The cycle is then repeated. The waveforms of the sensor are depicted in Fig. 3. Because of the positive feedback and the very high gain of the comparator, the output is a square wave and the oscillation of the chip temperature is approximately a triangular wave. The jump of $V_{1}$ is caused by the hysteresis of the feedback resistor $\mathbf{R}_{\mathrm{f}}$. The temperature oscillation amplitude can be chosen to be sufficiently small. Therefore, the average temperature $T_{\text {ave }}$ is almost constant.

The average heating power can therefore be written as

$P_{\text {ave }}=P_{\max } \frac{t_{\mathrm{r}}}{t_{\mathrm{p}}}=A_{\mathrm{c}} h\left(T_{\text {ave }}-T_{\mathrm{a}}\right)$

where $t_{\mathrm{r}} / t_{\mathrm{p}}$ is the duty cycle. Equation (7) shows that the duty-cycle output is proportional to the heating power and therefore to the heat-transfer coefficient.

\subsection{Thermal Oscillator with Positive Feedback} Synchronized by an External Clock

In the previous basic configuration, the oscillation frequency of the thermal oscillator depends
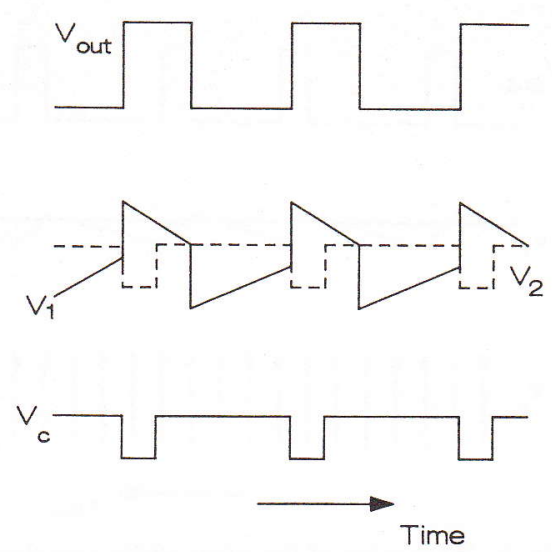

Fig. 4. Waveforms of the oscillator with an external clock.

mainly on the thermal capacitance of the chip and the transition region of the comparator. The oscillation frequency of the oscillator can be synchronized by an external clock in a certain range when this signal is added to the sensor signal, as depicted in Fig. 2. The waveforms of the thermal oscillator with positive feedback synchronized by an external clock are depicted in Fig. 4.

\subsection{Sigma-Delta Thermal Modulator}

Sensors with direct digital signal output are becoming more and more important in present microprocessor-based measurement systems. A very popular analog-to-digital converter is the sigma-delta modulator, which can also be extended to the thermal domain. This can easily be done by inserting a clocked master/slave flip-flop between the comparator and the heating resistor (see Fig. 5). This combination performs a one bit D/A conversion into the thermal domain by producing a heat pulse of one clock period. The

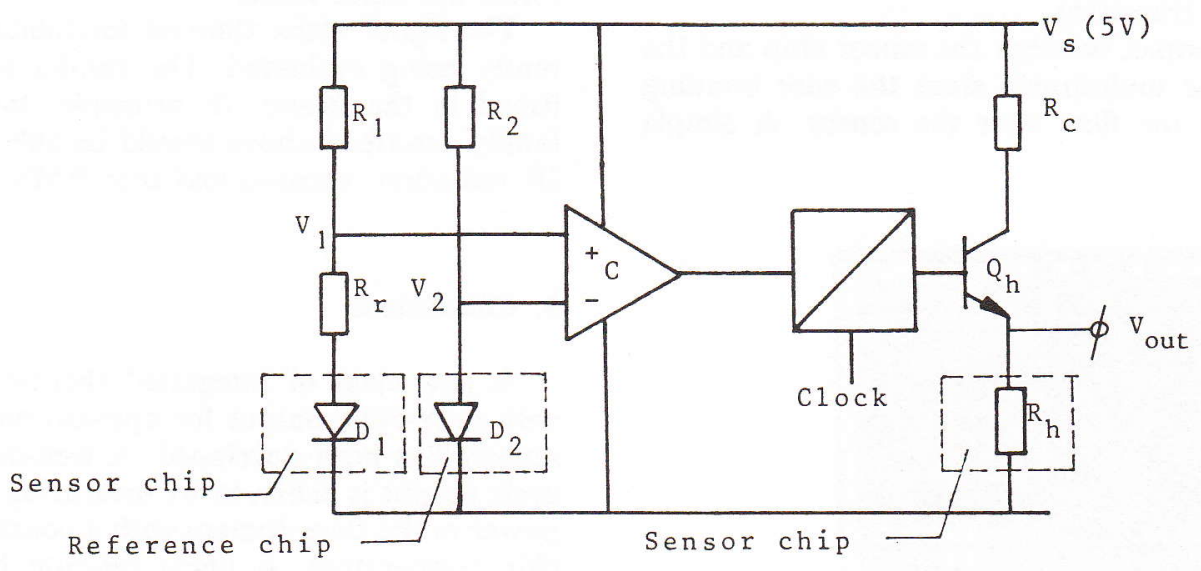

Fig. 5. Sigma-delta thermal modulators. 


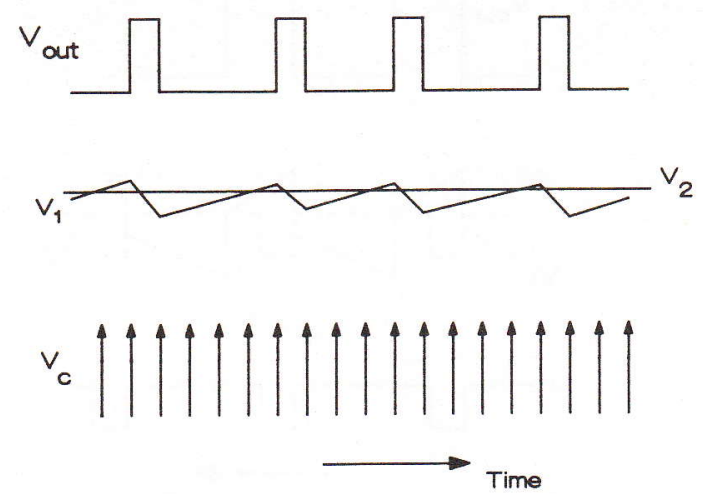

Fig. 6. Waveforms of the sigma-delta modulators.

thermal capacitance of the chip now serves as the integrating loop filter. The average rate of the heat pulses is proportional to the heating power, which is again related to the flow by eqn. (6). The waveforms are depicted by Fig. 6. By using a simple digital low-pass filter like a counter, a fully digital signal can be obtained. This signal can be transmitted by a bus which is connected to a microprocessor.

\section{Experimental Results}

The thermal converter family has been utilised to measure gas-flow velocity. A photograph of the integrated silicon flow sensor is depicted in Fig. 7. The chip measures $4 \times 3 \mathrm{~mm}$ and was processed by a standard bipolar IC process. It contains a transistor for measuring the on-chip temperature, a DP resistor for heating the chip, and a DPdiffusion Seebeck sensor (which is not used in our configuration). The sensor temperature is measured using the temperature dependence of the ' $V_{\text {be }}$ ' of the transistor.

Direct contact between the sensor chip and the fluid may be undesirable since the wire bonding will disturb the flow over the sensor. A simple

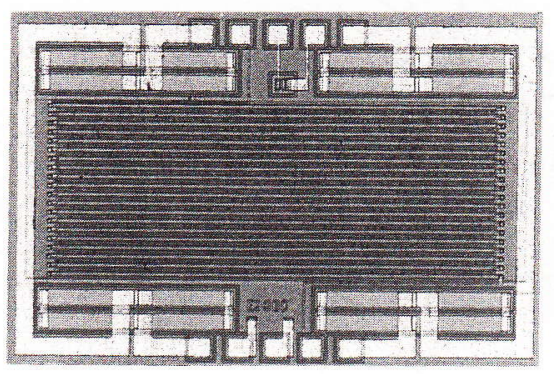

Fig. 7. Photograph of the sensor chip.

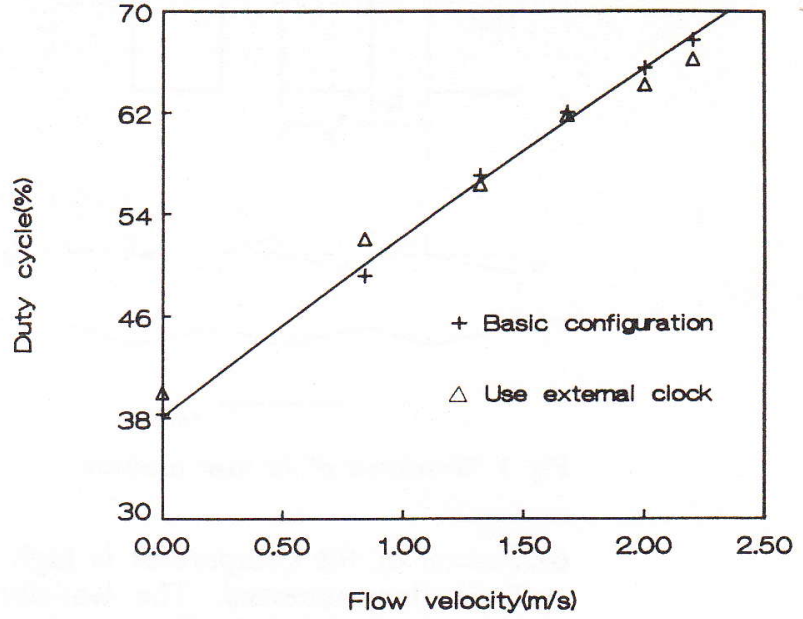

Fig. 8. Measured duty cycle and on-state probability as a function of flow velocity.

configuration was obtained by mounting the sensor chip onto a ceramic $\mathrm{Al}_{2} \mathrm{O}_{3}$ substrate carrying a gold interconnection pattern. This assembly is mounted upside down in a printed board which is placed in the mouth of a small wind tunnel. The sensor was operated at an overheat $10 \mathrm{~K}$ (emitter/ base offset $V_{\text {be }}=25 \mathrm{mV}$ ). The power supply was $5 \mathrm{~V}$.

The measured duty cycle as a function of the gas-flow velocity is depicted in Fig. 8. For the free-running thermal oscillators with positive feedback (with or without the external trigger signal), the oscillation of the chip temperature is a triangular wave with an amplitude of about $1 \mathrm{~K}$. The average sensitivity is $3.16 / \mathrm{mW} / \mathrm{K}$. With a block wave as a trigger signal, the output frequency of the oscillator can be selected from $20 \mathrm{~Hz}$ to $800 \mathrm{KHz}$ in our experiments. Moreover, the waveforms are more stable.

The sigma-delta thermal modulators are currently being evaluated. The results will be published in the future. In principle, the converter family described above should be able to measure IR radiation, vacuum and true RMS [7].

\section{Conclusions}

A new class of integrated thermal oscillators with duty-cycle output for application in thermal sensors has been developed. A well-defined dutycycle output is achieved by measuring the heating power in the time domain with a constant average chip temperature. A linear relation between the heating power and heat-transfer coefficient is obtained and the response time is greatly reduced. 
Several different structures can be utilised to construct thermal oscillators with different characteristics. Some experiment results for measuring flow velocity are presented. The principle can also be used to measure the vacuum, IR radiation and a true RMS.

The principle of the measurement has been well established, while the accuracy should be improved further. The power dissipation by a heating resistor is not a very accurate quantity in a standard bipolar technique. Moreover, the fluctuation of the power supply will further also reduce the accuracy of on-chip power.

Future work will concentrate on constructing an accurate on-chip heating source and on integrating all external elements on one single chip leading to the so-called 'smart sensors'.

\section{References}

1 J. H. Huijsing, J. P. Schuddemat and W. Verhoef, Monolithic integrated direction-sensitive flow sensor, IEEE Trans. Electron Devices, ED-29 (1982) 133-136.

2 O. Tabata, Fast-response silicon flow sensor with on-chip fluid temperature sensitive element, IEEE Trans. Electron Devices ED-33 (1986) 361-365.

3 G. E. Plstger and M. Southfield, Solid-state fluid sensor, U.S. Patent 3992940 (Nov. 23, 1976).

4 Tong Qin-Yi and Huang Jin-Biao, A novel CMOS flow sensor with constant chip temperature (CCT) operation, Sensors and Actuators, 12 (1988) 9-21.

5 G. Stemme, A CMOS integrated silicon gas flow sensor with pulse-modulation output, Proc. 4th Int. Conf. Solid-State Sensors and Actuators (Transducer '87), Tokyo, Japan, June $2-5,1987$, pp. $364-367$

6 Y. Pan and J. H. Huijsing, New integrated gas-flow sensor with duty-cycle output, Electron. Lett., 24 (1988) 542-543.

7 A. W. Van Herwaarden and P. M. Sarro, Thermal sensors based on Seebeck effects, Sensors and Actuators, 10 (1986) $321-346$. 